Mots. Les langages du politique

Proximité

\title{
Vingt-cinq ans au service de l'étude des langages du politique
}

Paul Bacot

\section{CpenEdition}

1 Journals

Édition électronique

URL : https://journals.openedition.org/mots/286

DOI : $10.4000 /$ mots.286

ISSN : 1960-6001

Éditeur

ENS Éditions

Édition imprimée

Date de publication : 1 mars 2005

Pagination : 3-4

ISBN : 2-84788-077-1

ISSN : 0243-6450

\section{Référence électronique}

Paul Bacot, "Vingt-cinq ans au service de l'étude des langages du politique », Mots. Les langages du politique [En ligne], 77 | 2005, mis en ligne le 31 janvier 2008, consulté le 23 avril 2022. URL : http:// journals.openedition.org/mots/286 ; DOI : https://doi.org/10.4000/mots.286 


\section{Vingt-cinq ans au service de l'étude des langages du politique}

Notre revue est vivante. Elle évolue. Elle tient compte des changements intervenant dans son environnement, qu'ils produisent des contraintes ou des opportunités nouvelles. Elle s'appuie sur ses acquis les plus solides que sont ses reconnaissances institutionnelles, son lectorat et ses collaborations. Elle est riche d'un passé vieux déjà d'un quart de siècle. Fondée en 1980 et longtemps dirigée par Maurice Tournier, elle bénéficie d'une expérience et d'une tradition qui sont des gages pour l'avenir.

Aujourd'hui, le changement de direction s'accompagne de quelques modifications dans la conception de la revue. La première sera le passage, dès l'an prochain, à une périodicité trimestrielle offrant chaque année quatre numéros et non plus seulement trois. La seconde sera, à la même date, la transition d'une formule presque exclusivement thématique vers la publication d'un dossier plus resserré accompagné d'une partie Varia rassemblant plusieurs articles portant sur des sujets différents. La troisième découle des deux premières : c'est la possibilité d'accueillir dès à présent des propositions d'articles en plus grand nombre et de les publier dans des délais plus brefs. Mais le vingt-cinquième anniversaire de la revue sera aussi marqué par l'ouverture, dans le courant de l'année, du site Internet qui lui fait encore défaut. Nous y reviendrons dans un prochain numéro.

Dès à présent, des améliorations sont apportées dans la présentation de la revue : nouveau format, nouvelle police de caractères. Mais plus profondément, ce sont l'organisation et les soutiens de la revue qui sont largement remaniés. Toujours éditée à Lyon par ENS Éditions, et dorénavant diffusée par les Presses universitaires de France, elle bénéficie de l'appui de trois équipes de recherche et d'une société savante. Il s'agit du CEDITEC (EA 3119, Université Paris 12), d'ICAR (UMR CNRS 5191, Université Lumière Lyon 2, ENS-LSH) et du nouveau laboratoire Triangle (UMR CNRS 5206, ENS-LSH, Université Lumière Lyon 2, Institut d'études politiques de Lyon). Ce trio marque bien le caractère pluridisciplinaire de la revue, qui se réclame principalement des sciences de l'information et de la communication, des sciences du langage et de la science politique. Sa nouvelle direction en est le reflet, puisque le directeur appartient à cette dernière discipline et les directeurs adjoints aux deux premières. Quant 
à la Société des amis de Mots (SAM), devenue Société d'étude des langages du politique (SELP), elle doit contribuer à fournir, notamment à travers ses journées d'étude comme celle qui, cette année, se tiendra en octobre à Toulouse, un environnement scientifique associant toutes celles et tous ceux qui, dans les différentes sciences humaines et sociales, ont fait des multiples formes de discours politiques un objet d'étude privilégié.

L'ancien comité de rédaction laisse la place à un comité éditorial et à un comité de lecture, tandis que le conseil scientifique est renouvelé dans l'optique de permettre à la revue de bénéficier plus largement de ses avis et de son expertise. Pierre Fiala a demandé à être déchargé de la direction mais reste très impliqué dans la nouvelle équipe. Qu'il soit remercié pour le travail qu'il a accompli et les résultats qu'il a obtenus, dans la continuité du chemin tracé par Maurice Tournier. Au-delà des deux directeurs successifs, que soient aussi remerciés celles et ceux qui ont donné beaucoup de leur temps et de leur énergie pour que vive notre revue, notamment Marie-Anne Paveau, qui en fut la directrice adjointe en une période décisive.

Paul Bacot 